\title{
Correlation between lipid profile and myeloperoxidase levels in type-2 diabetes mellitus
}

\author{
Sujatha $\mathbf{R}^{1}$, Gayathri $\mathbf{B}^{2}$ \\ ${ }^{1}$ Dr. Sujatha R Assistant Professor, ${ }^{2}$ Dr. Gayathri B, Professor, all authors are affiliated with Department of \\ Biochemistry, PSGIMSR, Coimbatore, Tamilnadu, India.
}

Address for Correspondence: Dr. Sujatha R, Email id: suja1357@gmail.com

\begin{abstract}
Introduction: Diabetes mellitus is the most common endocrine disorder caused by absolute or relative deficiency of insulin. Chronic hyperglycemia and enhanced oxidative stress have been implicated in the pathogenesis of diabetic vascular complications. These patients are more prone to adverse cardiovascular events which occur due to the accelerated rate of atherosclerosis in diabetic patients. Myeloperoxidase apart from playing a major role in innate immunity has been implicated in the various stages of atherosclerosis namely initiation, progression and plaque rupture. The objective of the study was to estimate the plasma myeloperoxidase activity in type-2 diabetics and to establish a correlation between plasma myeloperoxidase activity and lipid profile and glycated haemoglobin $\left(\mathrm{HbA}_{1} \mathrm{c}\right)$ in the study population. Methods: Plasma myeloperoxidase activity was estimated by spectrophotometric method using O-dianisidine dihydrochloride as substrate. Lipid profiles, $\mathrm{HbA}_{1} \mathrm{c}$ and blood sugar was analysed in an auto- analyser. Results: There was a statistically significant increase in plasma myeloperoxidase activity in diabetic patients when compared with non-diabetic individuals $(p<0.001)$. There is a significant positive correlation between plasma myeloperoxidase activity and serum triglyceride levels in the study population ( $\mathrm{p}<0.001$ ). Conclusion: Plasma MPO activity was significantly higher in the diabetic patients when compared with nondiabetic controls. There is significant positive correlation between MPO activity and serum triglyceride levels.
\end{abstract}

Keywords: Diabetes mellitus, Atherosclerosis, Myeloperoxidase, Cardiovascular Disease, Lipid profile.

\section{Introduction}

Diabetes mellitus (DM) is a metabolic disorder, which occupies the seventh leading cause of death [1]. The burden caused by the morbidity and mortality in patients with type-2 DM is mainly driven by its vascular manifestations. DM is associated with increased risk of premature vascular disease due to accelerated atherosclerosis [2].

Myeloperoxidase (MPO), an enzyme of the heme peroxidase superfamily in mammals is present within the azurophilic granules of leukocytes. It plays a major role in innate host defense by participating in the oxygen dependent killing of invading pathogens [3].

MPO is capable of generating reactive oxygen species that modify lipids and proteins which contributes to

Manuscript received $10^{\text {th }}$ July 2016

Reviewed: $24^{\text {th }}$ July 2016

Author Corrected: $7^{\text {th }}$ August 2016

Accepted for Publication 24 $4^{\text {th }}$ August 2016 atherogenesis. Recent studies have shown that low density lipoprotein (LDL) on exposure to reactive nitrogen and halogen species by MPO gets converted into more atherogenic forms in the walls of the blood vessel [4]. Also MPO participates in the generation of dysfunctional high density lipoprotein (HDL) contributing to pro-inflammatory state which triggers atherosclerosis [5]. Apart from this, MPO consumes endothelial derived nitric oxide, thus reducing its bioavailability and impairing its functions such as vasodilatation and anti-inflammatory properties [4]. Thus MPO is found to contribute to endothelial dysfunction, the major pathogenetic mechanism in the process of atherosclerosis.

It is well known that endothelial dysfunction develops early in DM, preceding clinically detectable atherosclerosis, and that diminished nitric oxide levels and enhanced oxidative stress are important factors in 
the pathogenesis of diabetic vascular complications [2]. MPO plays an important role in the initiation, progression and the complications of atherosclerosis. Previous studies have specifically focused on the relation between MPO levels and the presence of endothelial dysfunction in diabetes $[6,7]$.

Evidence regarding MPO activity in diabetes is scarce and contradictory, indicating both higher and lower levels of MPO in different tissues clinical situations [6, 7].

These studies showed a significantly higher MPO levels in diabetes. But some studies also showed no correlation of MPO level in diabetes [8]. Not many works are there to see the relationship between MPO and $\mathrm{HbA} 1 \mathrm{c}$ and also with lipid abnormalities. Hence this study is undertaken to estimate the plasma MPO activity, lipid profile and $\mathrm{HbA} 1 \mathrm{c}$ and also to observe the relationship between them.

\section{Materials and Methods}

After obtaining ethical clearance from the Institutional Human Ethics Committee, the study was conducted for a period of one year in a tertiary care hospital. An informed consent was taken from the patients before sample collection.

It is a cross-sectional study involving two groups. Nondiabetic healthy individuals who were attending the master health check-up served as controls $(n=50)$. The cases included patients from the out-patient department of medicine and endocrinology diagnosed with type-2
DM as per WHO criteria not less than one year duration. $(n=50)$. Age and gender matched participants were selected randomly and included in the study.

Diabetic patients with vascular complications, patients with auto-immune disease, hypertension, and patients on treatment with statins were excluded from the study. $5 \mathrm{ml}$ of venous blood sample was collected after a period of 12 hours overnight fasting. HbA1c, lipid profile and plasma MPO activity were estimated in the sample. Anthropometric measurements like weight and height were also recorded.

Glycated hemoglobin was measured by Turbidimetric inhibition immunoassay in Cobas Integra 400 autoanalyzer. Lipid profile parameters were analyzed using enzymatic methods in Cobas Integra 400 auto-analyzer using dedicated kits and reagents.

Plasma MPO was estimated by spectrophotometric method using O-dianisidine dihydrochloride as a substrate [9].

Statistical Analysis: Baseline characteristics are measured as Mean \pm SD. Significance is assessed at $5 \%$ level of significance. Student ' $t$ ' test (two tailed, independent) and Chi-square test has been used to find the significance of study parameters. ROC curve analysis is performed to find the sensitivity and specificity of the diagnostic test (to be deleted). Pearson correlation was done to establish correlation between MPO and other relevant parameters such as lipid profile and $\mathrm{HbA} 1 \mathrm{c}$.

\section{Results}

The mean age of the control group was $48.12 \pm 8$.56years and that of diabetic group were $51.30 \pm 11.41$ years. The mean BMI in controls were $26.4 \pm 4.4 \mathrm{~kg} / \mathrm{m}^{2}$ and in diabetic patients were $27.06 \pm 4.49 \mathrm{~kg} / \mathrm{m}^{2}$ which had no statistical significant difference between the study groups. The fasting blood sugar levels were significantly higher in the diabetic population. The mean blood glucose levels in the control group are $93.14 \pm 8.15 \mathrm{mg} / \mathrm{dL}$ and it is $204.14 \pm 75.64 \mathrm{mg} / \mathrm{dL}$ in the diabetic cases group.

There was statistically significant difference in the triglyceride levels of the diabetic cases when compared with the nondiabetic controls. The mean triglyceride concentration in the control groups were $128.38 \pm 53.19 \mathrm{mg} / \mathrm{dL}$ and that in diabetic group was $164.16 \pm 90.55 \mathrm{mg} / \mathrm{dL}$ [p value 0.02]. The other parameters of the lipid profile did not differ significantly between the two study groups [Table 1]. The levels of serum myeloperoxidase was estimated and compared in the study groups [Graph 1]. The mean values in the controls and cases group were 188.41 $\pm 142.73 \mathrm{U} / \mathrm{L}$ and $317.17 \pm 247.73 \mathrm{U} / \mathrm{L}$ respectively.

Correlational analysis between myeloperoxidase activity and BMI [expressed as $\mathrm{kg} / \mathrm{m}^{2}$ ], serum cholesterol [expressed as $\mathrm{mg} / \mathrm{dL}$ ], serum triglyceride [expressed as $\mathrm{mg} / \mathrm{dL}$ ], serum HDL [expressed as $\mathrm{mg} / \mathrm{dL}$ ], glycated hemoglobin [measured as $\%]$ in the diabetic patients was done using Pearson's correlational testing. 
The Pearson correlation coefficients of myeloperoxidase activity with total cholesterol, triglyceride, BMI and glycated hemoglobin is shown in [Table 2].

Table-1: Comparison of lipid profile between the study groups

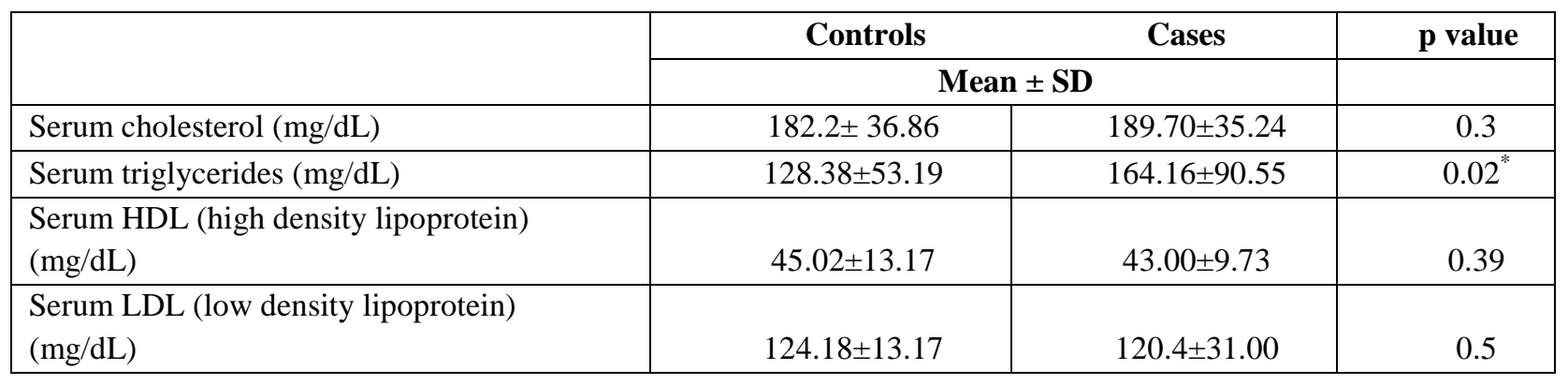

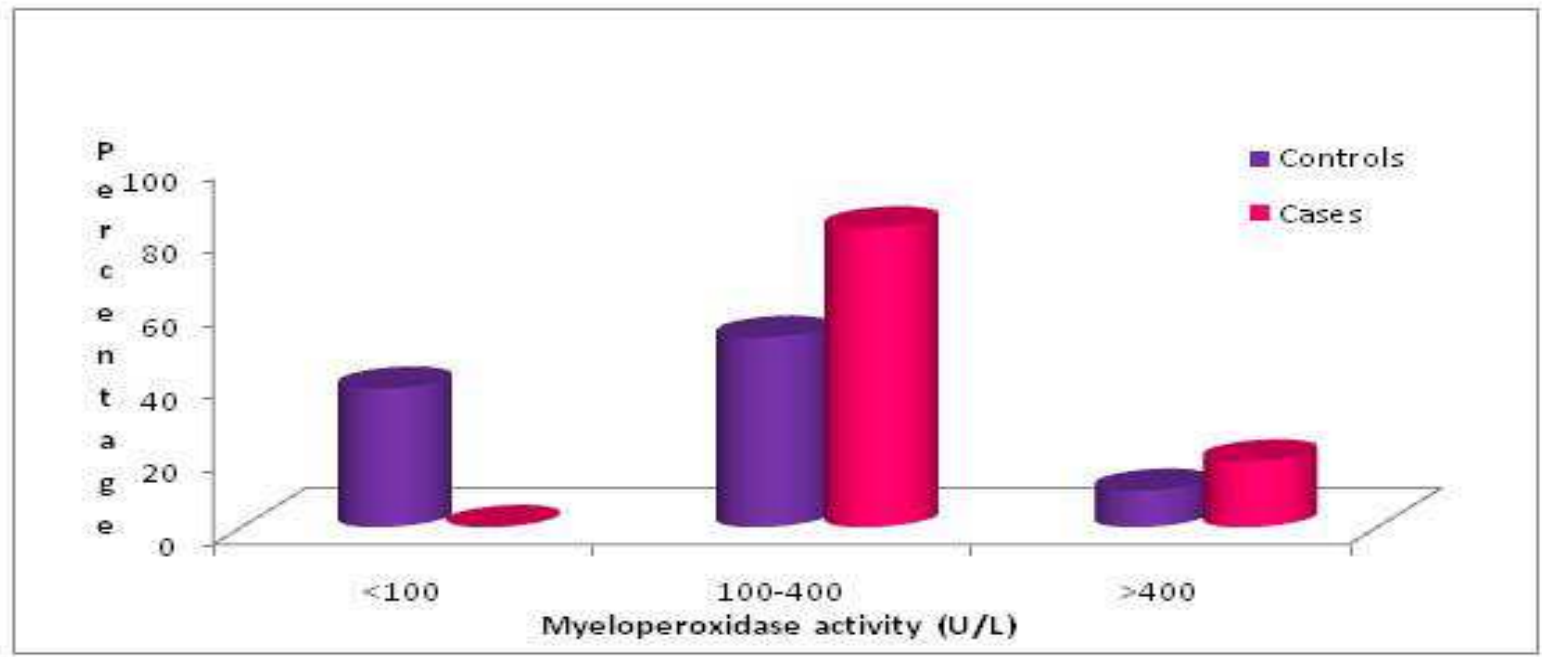

Graph-1: Myeloperoxidase activity in the study population

Table 2: Pearson's correlation analysis between MPO activity and BMI, Serum Cholesterol, Serum Triglycerides, Serum HDL, HbA1c.

\begin{tabular}{|l|c|c|}
\hline & r value & p value \\
\hline MPO activity \& BMI & 0.154 & 0.286 \\
\hline MPO activity \& Serum Cholesterol & 0.272 & 0.056 \\
\hline MPO activity \& Serum HDL & -0.091 & 0.345 \\
\hline MPO activity \& Serum Triglycerides & 0.437 & $0.002^{*}$ \\
\hline MPO activity \& HbA1c & 0.235 & 0.1 \\
\hline
\end{tabular}

In the present study, a significant positive correlation is observed between myeloperoxidase activity and serum triglyceride levels.

\section{Discussion}

Diabetes mellitus has developed as a disease of major social and economic concern due to the development of its vascular complications. Cardiovascular disease is the most common cause of morbidity and mortality in diabetic patients. Early detection and identification of the cardiovascular complications can lead to a decrease in the burden of the disease.
Myeloperoxidase is a heme containing enzyme which plays a major role in innate host defense by participating in the oxygen dependent killing of invading pathogens. A considerable number of epidemiological and clinical studies have demonstrated an association between increased concentrations of myeloperoxidase and cardiovascular disease, 
independent of classical risk factors. Myeloperoxidase activity leads to oxidation of LDL cholesterol, which increases its atherogenicity. Myeloperoxidase -induced oxidation of HDL cholesterol can reduce its capacity for reverse cholesterol transport. Myeloperoxidase activity leads to consumption of endothelial derived nitric oxide, which can lead to plaque formation and endothelial dysfunction. As a result of its implication in the development of atherosclerosis, myeloperoxidase has been established as a marker of cardiovascular disease [4]. This study was done to estimate the levels of Myeloperoxidase in diabetic and non-diabetic patients and compare it in both the groups.

In the present study, plasma Myeloperoxidase levels were significantly higher in the diabetic patients when compared to the non-diabetic controls [p <0.05]. This finding was consistent with the study by Wiersma et al [10] in which there were a significant increase in the Myeloperoxidase levels of diabetic patients. DM is considered to be a state of low grade and chronic inflammation. The development of endothelial dysfunction in diabetic patients makes them more prone to acceleration in the process of atherosclerosis. This predisposes these individuals to increased risk of cardiovascular disease. Myeloperoxidase has been established as a marker for cardiovascular disease. It has been implicated in the evolution of atherosclerosis by leading to the formation of atherogenic lipoproteins such as oxidation of LDL, defective function of HDL and also consumption of endothelial nitric oxide [4]. This explains the increase in Myeloperoxidase activity in diabetic patients.

The fasting plasma glucose levels were significantly higher in the diabetic population compared to the nondiabetic group. This further can explain the increase in Myeloperoxidase activity in diabetic patients. High glucose stimulates the production of hydrogen peroxide. This hydrogen peroxide which is a physiological substrate for Myeloperoxidase is converted to hypochlorous acid. Thus high glucose results in increase in Myeloperoxidase activity [11].

Plasma Myeloperoxidase activity in both the groups is found to increase with an increase in body mass index. Myeloperoxidase activity of $>400 \mathrm{U} / \mathrm{L}$ is more in the controls and cases with body mass index of $\geq 30 \mathrm{~kg} / \mathrm{m}^{2}$. The association of obesity with inflammation and cardiovascular risk is a possible explanation for the increase in Myeloperoxidase activity. When there is excess supply of nutrients such as glucose, expanding adipocytes present oxidative stress in the endoplasmic reticulum which is an important trigger for the different inflammatory kinases such as JNK [Jun N-terminal Kinase] and IKK [Inhibitor of Kappa B Kinase]. This can eventually inhibit insulin signaling and activate inflammatory pathways [12].

There is significant increase in the serum triglyceride levels in the diabetic population when compared to the non-diabetic subjects [p value of 0.02]. This increase in triglyceride levels in diabetic patients can be attributed to the inflammation evident in diabetes mellitus. The inflammatory mediators like the cytokines increase the synthesis of VLDL by increased fatty acid synthesis in the liver. They also reduce VLDL (very low density lipoprotein) clearance by decrease in lipoprotein lipase and hepatic lipase activity which in turn leads to elevated triglyceride levels [13].

The other common lipid abnormalities in diabetes mellitus are decrease in HDL and increase in the levels of LDL which has been attributed to the hyperglycemia. In this study, there were no statistically significant changes in the concentration of HDL and LDL. But it has been reported that myeloperoxidase has been found to produce dysfunctional HDL. In this study the function of HDL has not been studied.

Pearson's correlational analysis revealed a statistically significant correlation between serum triglyceride levels and myeloperoxidase activity in diabetic patients. The link between hypertriglyceridemia and increased myeloperoxidase activity can be attributed to inflammation. The non-esterified fatty acids released from the excess triglycerides are pro-inflammatory and they activate the inflammatory mediators which ultimately lead to an inflammatory state [14].

\section{Conclusion}

DM is an inflammatory condition which is characterised by chronic hyperglycemia. The increased glucose levels leads to the development of vascular complications in DM. Several markers have been established to identify the vascular disturbances in these patients at an earlier stage.

MPO has been implicated in the development of all stages of atherosclerosis by promoting LDL oxidation, producing dysfunctional HDL and reducing nitric oxide bioavailability. 
In the present study

$\checkmark$ Plasma MPO activity was significantly higher in the diabetic patients when compared with non-diabetic controls.

$\checkmark$ There is significant positive correlation between MPO activity and serum triglyceride levels.

Funding: Nil, Conflict of interest: None initiated, Permission from IRB: Yes

\section{References}

1. National Vital Statistics Report. Deaths: Leading causes for 2010. NVSR Vol.62, number 6. 2014-1120.

2. Hartge MM, Unger T, Kintscher U. The endothelium and vascular inflammation in diabetes. Diabetes and Vascular Disease Research. 2007 Jun 1;4(2):84-8.

3. Klebanoff SJ. Myeloperoxidase: friend and foe. Journal of leukocyte biology. 2005 May 1;77(5):598625.

4. Nicholls SJ, Hazen SL. Myeloperoxidase, modified lipoproteins, and atherogenesis. Journal of lipid research. 2009 Apr 1;50(Supplement):S346-51.

5. Vaisar T, Shao B, Green PS, Oda MN, Oram JF, Heinecke JW. Myeloperoxidase and inflammatory proteins: pathways for generating dysfunctional highdensity lipoprotein in humans. Current atherosclerosis reports. 2007 Nov 1;9(5):417-24.

6. Böker T, Augustin AJ, Breipohl W, Spitznas M, Lutz J. Increased lipid peroxide level and myeloperoxidase activity in the vitreous of patients suffering from proliferative vitreoretinopathy. Graefe's archive for clinical and experimental ophthalmology. 1994 Nov 1;232(11):652-6.

7. Sato N, Shimizu H, Suwa K, Shimomura Y, Kobayashi I, Mori M. MPO activity and generation of active $\mathrm{O} 2$ species in leukocytes from poorly controlled diabetic patients. Diabetes Care. 1992 Aug 1;15(8): 1050-2.
8. Shetty S, Kumari SN, Madhu LN. Variations in Serum Myeloperoxidase Levels With Respect To Hyperglycemia, Duration of Diabetes, BMI, Sex And Aging in Type 2 Diabetes Mellitus. Int J Res Pharm Biomed Sci. 2012 Apr;3(2):652-5.

9. Bradley PP, Priebat DA, Christensen RD, Rothstein G. Measurement of cutaneous inflammation: estimation of neutrophil content with an enzyme marker. Journal of Investigative Dermatology. 1982 Mar 31;78(3):206-9.

10. Wiersma JJ, Meuwese MC, van Miert JN, Kastelein A, Tijssen JG, Piek JJ, Trip MD. Diabetes mellitus type 2 is associated with higher levels of myeloperoxidase. Medical science monitor. 2008 Aug 1;14(8):CR406-10.

11. Zhang C, Yang J, Jennings LK. Leukocyte-derived myeloperoxidase amplifies high-glucose-induced endothelial dysfunction through interaction with highglucose-stimulated, vascular non-leukocyte-derived reactive oxygen species. Diabetes. 2004 Nov 1;53 (11):2950-9.

12. Olza J, Aguilera CM, Gil-Campos M, Leis R, Bueno G, Martínez-Jiménez MD, Valle M, Cañete R, Tojo R, Moreno LA, Gil A. Myeloperoxidase is an early biomarker of inflammation and cardiovascular risk in prepubertal obese children. Diabetes care. 2012 Nov 1;35(11):2373-6.

13. Moldoveanu E, Tanaseanu C, Tanaseanu S, Kosaka T, Manea G, Marta DS, Popescu LM. Plasma markers of endothelial dysfunction in type 2 diabetics. European journal of internal medicine. 2006 Jan 31;17(1):38-42.

14. Sarwar N, Danesh J, Eiriksdottir G, Sigurdsson G, Wareham N, Bingham S, Boekholdt SM, Khaw KT, Gudnason V. Triglycerides and the risk of coronary heart disease 10158 incident cases among 262525 participants in 29 western prospective studies. Circulation. 2007 Jan 30;115(4):450-8.

\section{How to cite this article?}

Sujatha R, Gayathri B. Correlation between lipid profile and myeloperoxidase levels in type-2 diabetes mellitus. Int J Med Res Rev 2016;4(9):1538-1542.doi:10.17511/ijmrr.2016.i09.04. 